\title{
FUNCTIONAL DIFFERENTIAL EQUATIONS CLOSE TO DIFFERENTIAL EQUATIONS
}

\author{
BY KENNETH L. COOKE ${ }^{1}$
}

Communicated by H. A. Antosiewicz, October 7, 1965

In a recent Research Problem [1], Bellman has raised the question of the behavior of solutions of the functional differential equation

$$
u^{\prime}(t)+a u(t-r(t))=0
$$

when the lag function $r(t)$ is nearly constant for large $t$, and has also asked for conditions on the function $r$ under which all solutions approach zero as $t \rightarrow \infty$. The purpose of this announcement is to initiate a study of various stability and oscillation problems for equations with perturbed lag functions, and to suggest that a modification of the familiar method of conversion to an integral equation can be profitably employed for these problems.

In this announcement, we characterize the asymptotic behavior of solutions of Equation (1) in case $r(t)$ tends to zero with a certain order as $t \rightarrow \infty$. We go beyond the problem posed by Bellman by establishing the asymptotic equivalence of Equation (1) and an approximating ordinary differential equation. In subsequent work, we shall deal with cases in which $r(t)$ is asymptotically constant, or is oscillatory, extensions to higher order equations, and so on. In particular, we shall deal with the remaining parts of Bellman's Research Problem. The first result obtained is as follows:

THEOREM 1. Let $r(t)$ be continuous and nonnegative for $t \geqq t_{0}$ and assume that the following conditions are satisfied:

$$
\begin{aligned}
\text { (i) } \quad r(t) & \rightarrow 0 \text { as } t \rightarrow+\infty \text {; } \\
\text { (ii) } \quad \int^{\infty} r(t) d t & <\infty ; \\
\text { (iii) } \inf _{t \geqq t_{0}}[t-r(t)] & >-\infty .
\end{aligned}
$$

Then every continuous solution of Equation (1) for $t \geqq t_{0}$ satisfies

$$
\lim _{t \rightarrow+\infty} u(t) e^{a t}=c
$$

for some constant c. Moreover, for every $c,-\infty<c<+\infty$, there is a

1 This work was partially supported by NSF Grant GP4029. 
solution of Equation (1) which satisfies (3).

Sketch of Proof. Details of the proof will be published elsewhere. The main features of the proof can be outlined as follows. We rewrite Equation (1) as

$$
u^{\prime}(t)+a u(t)=a[u(t)-u(t-r(t))], \quad t>t_{0},
$$

and treat the right member as a forcing term. Every solution of Equation (4) on $t>t_{0}$ satisfies

$$
u(t) e^{a t}=u\left(t_{0}\right) e^{a t_{0}}+a \int_{t_{0}}^{t} e^{a t_{1}}\left[u\left(t_{1}\right)-u\left(t_{1}-r\left(t_{1}\right)\right)\right] d t_{1} .
$$

Observe that there is a constant $c_{1}$, which can be taken independent of $t_{0}$, such that

$$
\left|1-e^{a r(t)}\right| \leqq c_{1} r(t), \quad t \geqq t_{0} .
$$

Let $\tau_{0}=\inf _{t \geq t_{0}}[t-r(t)]$ and for convenience let $c_{0}=u\left(t_{0}\right) e^{a t_{0}}$. Let $g(t)$ denote any fixed but arbitrary continuous function defined on $\tau_{0} \leqq t \leqq t_{0}$. This function will be regarded as an "initial function" for a solution of Equation (1). We show that to each such $g$, there is a unique solution of Equation (1) lying in a certain class $S_{b}$ defined in the following way. Choose any number $b$ satisfying

$$
\begin{aligned}
& b>4\left|c_{0}\right| c_{1}, \\
& b>\max \left\{|g(t)| e^{a t}: \tau_{0} \leqq t \leqq t_{0}\right\} .
\end{aligned}
$$

Let $\tau_{1}$ be the first number greater than $t_{0}$ such that $\tau_{1}-r\left(\tau_{1}\right)=t_{0}$ and $t-r(t)>t_{0}$ for $t>\tau_{1}$.

Define $S_{b}$ as the class of continuous functions $u$ defined for $t \geqq \tau_{0}$ and satisfying

$$
\begin{aligned}
u(t) & =g(t), & & \tau_{0} \leqq t \leqq t_{0} \\
|u(t)| e^{a t} & \leqq b, & & \tau_{0} \leqq t, \\
|u(t)-u(t-r(t))| e^{a t} & =c_{2} b r(t), & & \tau_{1} \leqq t,
\end{aligned}
$$

where $c_{2}$ is a certain fixed constant depending on $a$ and $r(t)$. We now consider the mapping $v=M u$ defined, for fixed $g$, by

$$
\begin{array}{rlrl}
v(t) & =g(t), & \tau_{0} \leqq t \leqq \\
v(t) e^{a t} & =c_{0}+a \int_{t_{0}}^{t} e^{a t_{1}}\left[u\left(t_{1}\right)-u\left(t_{1}-r\left(t_{1}\right)\right)\right] d t_{1}, & & t \geqq t_{0},
\end{array}
$$

with $c_{0}=g\left(t_{0}\right) e^{a t_{0}}$. The existence of a fixed point of this mapping, lying 
in the set $S_{b}$, can be established by using the Tychonoff fixed point theorem in a manner similar to that of Stokes, [2], [3].

Following Stokes, we take for $E$ the real linear space of continuous functions from $\left[\tau_{0}, \infty\right)$ into $R^{n}$, with the compact-open topology. It can be shown that if $c_{2}$ is suitably chosen and if $t_{0}$ is chosen large enough then $S_{b}$ is convex, closed, and bounded in this topology, and that $M$ maps $S_{b}$ into itself and is completely continuous. Since $E$ is a metric space, $M\left(S_{b}\right)$ is compact in $E$ and $\mathrm{Cl}\left[M\left(S_{b}\right)\right]$ is compact. It therefore follows from the Tychonoff theorem that there is a fixed point of $M$ lying in the set $S_{b}$. It is readily seen that this fixed point is a solution of Equation (1) for $t>t_{0}$ satisfying $u(t)=g(t)$ for $\tau_{0} \leqq t \leqq t_{0}$ and satisfying the inequalities in (7). Since there is a unique solution of Equation (1) with given $g$, every solution of Equation (1) must satisfy the inequalities in (7).

Using the second inequality in (7), and the hypothesis on $r$, we easily see that $u(t) e^{a t} \rightarrow c$ as $t \rightarrow \infty$ where

$$
c=g\left(t_{0}\right) e^{a t_{0}}+a \int_{t_{0}}^{\infty} e^{a t_{1}}\left[u\left(t_{1}\right)-u\left(t_{1}-r\left(t_{1}\right)\right)\right] d t_{1} .
$$

Thus, every solution satisfies (3) for some $c$. By choosing $g(t)$ in a special way, it is possible to find one solution for which $c \neq 0$. Then, since any multiple of a solution is a solution, there is a solution satisfying (3) for every $c,-\infty<c<+\infty$.

Asymptotic series. If $r(t)$ has an asymptotic power series expansion, the equation

$$
u(t) e^{a t}=c-a \int_{t}^{\infty} e^{a t_{1}}\left[u\left(t_{1}\right)-u\left(t_{1}-r\left(t_{1}\right)\right)\right] d t_{1}
$$

can be used to prove that $u(t)$ does also.

Weaker hypothesis. If $r(t)=O\left(t^{-1}\right)$ but the condition (ii) in (2) is not satisfied, it is now necessary to work with a different integral equation. Heuristic arguments lead to the equation

$$
u(t) e^{s(t)}=u\left(t_{0}\right)+\int_{t_{0}}^{t} e^{s\left(t_{1}\right)}\left[\lambda\left(t_{1}\right) u\left(t_{1}\right)-a u\left(t_{1}-r\left(t_{1}\right)\right)\right] d t_{1}, \quad t>t_{0},
$$

where

$$
\begin{aligned}
& \lambda(t)=a+a^{2} r(t), \\
& s(t)=\int_{t_{0}}^{t} \lambda(\tau) d \tau .
\end{aligned}
$$

Arguments of the same general character as before, but more complicated, now lead to the following theorem. 
THEOREM 2. Let $r(t)$ be continuous and nonnegative for $t \geqq t_{0}$ and assume that the following conditions are satisfied:

(i) $r(t) \rightarrow 0$ and $r^{\prime}(t)$ is bounded as $t \rightarrow+\infty$;

(ii) $\inf _{t \geqq t_{0}}[t-r(t)]>-\infty$.

Then there is a constant $c_{1}$ independent of $t_{0}$ such that

$$
|\lambda(t)-a \exp [s(t)-s(t-r(t))]| \leqq c_{1} r(t)^{2}, \quad t \geqq t_{0} .
$$

Also assume that: (iii) for some $\delta>0$,

$$
\int^{\infty}[r(t)]^{2-\delta} d t<\infty
$$

(iv) if $\tau_{1}=\tau_{1}\left(t_{0}\right)$ is defined by

$$
\tau_{1}-r\left(\tau_{1}\right)=t_{0}, \quad t-r(t)>t_{0} \text { for } t>\tau_{1},
$$

then for $t_{0}$ sufficiently large

$$
\begin{array}{r}
8|a| \int_{\tau_{1}}^{t} r\left(t_{1}\right) d t_{1} \leqq r(t) \quad \text { for } \tau_{1} \leqq t, t-r(t) \leqq \tau_{1}, \\
4 c_{1} \int_{\tau_{1}}^{t} r\left(t_{1}\right) d t_{1} \leqq r(t)^{-\delta} \quad \text { for } \tau_{1} \leqq t, \tau_{1} \leqq t-r(t), \\
8|a| \int_{t-r(t)}^{t} r\left(t_{1}\right) d t_{1} \leqq r(t)^{2-\delta}, \quad t \geqq t_{0} .
\end{array}
$$

Then every solution of Equation (1) for $t \geqq t_{0}$ satisfies

$$
\lim _{t \rightarrow+\infty} u(t) e^{s(t)}=c
$$

for some constant $c$. Moreover, for every $c,-\infty<c<+\infty$, there is a solution of Equation (1) which satisfies this limit.

The conditions in (iv) are satisfied, for example, if $r(t)=t^{-1}$.

In conclusion we mention that a similar technique can be used if in Equation (1) the constant $a$ is replaced by $a+a(t)$ where $a(t) \rightarrow 0$.

\section{REFERENCES}

1. R. Bellman, Research problem: Functional differential equations, Bull. Amer. Math. Soc 71 (1965), 495.

2. A. Stokes, The applications of a fixed point theorem to a variety of non-linear stability problems, Contributions to the Theory of Nonlinear Oscillations, Vol. 5, pp. 173-184, Princeton Univ. Press, Princeton, N. J., 1960.

3. - Functional analysis and some fixed point problems, Dissertation, Univ. of Notre Dame, Notre Dame, Ind., 1958.

Pomona College 